\title{
Gemifloxacin use in the treatment of acute bacterial exacerbation of chronic bronchitis
}

This article was published in the following Dove Press journal:

International Journal of COPD

30 July 2009

Number of times this article has been viewed

\author{
Cristian Jivcu' \\ Mark Gotfried ${ }^{2}$ \\ 'Department of Internal Medicine, \\ Banner Good Samaritan Medical \\ Center, Phoenix, AZ, USA; \\ ${ }^{2}$ Department of Medicine, University \\ of Arizona, Phoenix, AZ, USA
}

\begin{abstract}
The newest generation of fluoroquinolones have proven efficacy against bacterial organisms associated with acute exacerbation of chronic bronchitis (AECB). Gemifloxacin, as one of the quinolones in this class, exhibits many of the pharmacokinetic and pharmacodynamic characteristics of the class with a few notable differences. Against Streptococcus pneumoniae it has a lower minimal inhibitory concentration (MIC) than the other respiratory fluoroquinolones and it has activity against both bacterial DNA gyrase and topoisomerase IV. The increased activity of gemifloxacin against both enzymes may be associated with decreased rates of resistance. Clinically, gemifloxacin has been shown to have positive effects on length of hospitalization and increased success at long-term follow-up in AECB patients. These associations were observed in noninferiority comparison studies. Although an advantage with the use of gemifloxacin in AECB is suggested, there are no comparison data is available to conclude that gemifloxacin is superior to the other respiratory fluoroquinolones. Gemifloxacin is generally well tolerated, but is associated with a characteristic rash and gastrointestinal upset as its most common observed side effects.
\end{abstract}

Keywords: gemifloxacin, respiratory fluoroquinolones, acute exacerbation of chronic bronchitis

\section{Introduction}

Chronic bronchitis is defined as daily cough and daily production of sputum for at least three consecutive months over two consecutive years. ${ }^{1}$ Most of the patients affected by this chronic condition also have underlying nonreversible airflow obstruction, or chronic obstructive pulmonary disease (COPD). In most cases the two conditions coexist. However, in some cases the chronic cough and sputum production may precede the development of airflow limitation. ${ }^{2}$ In other cases, significant airflow limitation may develop without the presence of bronchitic symptoms. ${ }^{1}$

Chronic bronchitis is generally a slowly progressive disease characterized by episodes of acute exacerbation. These exacerbations tend to become more frequent and more severe as the underlying disease advances leading to increased morbidity. The characteristic symptoms during these episodes are cough, dyspnea and changes in sputum volume and purulence. The number of symptoms that are present along with the patient's underlying pulmonary and comorbid diseases help in the classification of exacerbating episodes. Most patients have between 1-4 acute exacerbations annually. ${ }^{3}$

The most common risk factors for exacerbation are cigarette smoking, advanced age, and low baseline lung function. ${ }^{2}$ It is a well-known fact that chronic bronchitis 
and COPD typically develop in long-time smokers as they approach middle or old age. These patients, in addition to their decreasing lung function, are also at risk for developing other comorbid conditions either related to smoking or advancing age. ${ }^{1}$ In a 1996 study, $25 \%$ of COPD patients over 65 in the Netherlands had two comorbid conditions and up to $17 \%$ of had three comorbid conditions. ${ }^{4}$

The direct correlation between acute exacerbations of chronic bronchitis (AECB) and more rapid, permanent airway damage is well established. The "vicious circle" of acute exacerbations leading to recurrent inflammation and eventually to permanent airway damage, which in turn leads to more frequent and more profound exacerbations has been postulated by Murphy and colleagues ${ }^{5}$ and later by Wilson. ${ }^{6}$ As more data on inflammatory biomarkers became available, the same idea was re-explored by Sethi and colleagues. ${ }^{7}$ Inflammatory factors such as interleukin-6 (IL-6), C-reactive protein, IL-8, and tumor necrosis factor- $\alpha$ have all been noted to be elevated during exacerbations. Some of these biomarkers, such as procalcitonin, may eventually be useful in diagnosis of bacterial causes of AECB in the future. ${ }^{8}$

Treatment of AECB is multifactorial and it normally begins with recommendations regarding lifestyle changes, the most important of which without a doubt is smoking cessation. Supportive treatment might consist of oxygen supplementation in hypoxic patients, removal of environmental irritants, and appropriate hydration. Pharmaceutical treatment includes inhaled $\beta$-agonists, anticholinergics, inhaled or systemic corticosteroids, and antibiotic therapy.

The optimal antibiotic therapy for AECB has been debated for many decades, and even today it generates considerable debate in the pulmonary community given that only select groups of patients have been shown to benefit from the therapy. A significant obstacle in achieving consensus has been the fact that both patients with stable COPD and those with acute exacerbations often grow the same organisms in cultures, and as a result it is difficult to determine the exact role of bacteria in the exacerbating events. Following Anthonisen and colleagues' seminal 1987 trial, which showed significant response to antibiotic therapy in the sickest patients, it has been widely agreed that antibiotics should not be withheld from these patients. ${ }^{9}$

Several medical organizations, including the Canadian Thoracic Society (CTS), Canadian Infectious Diseases Society (CIDS), and in the US the Primary Care Consensus Guidelines among others, ${ }^{10,11}$ published treatment recommendations for AECB. Among the different recommendations there is agreement that patients who only have acute tracheobronchitis without underlying lung disease should be treated supportively without the addition of antibiotics. There are differences, however, concerning recommended treatment regimens and stratification criteria for patients with more severe symptoms, or underlying pulmonary disease. Anthonisen's criteria were based on the number of symptoms the patients have, while other criteria proposed by other authors also take into consideration the patient's underlying lung disease and comorbidities. ${ }^{12}$

Infectious agents are thought to cause $80 \%$ of exacerbations. Of these $50 \%-70 \%$ are estimated to be caused by bacterial agents and $30 \%-50 \%$ by viral agents. ${ }^{13}$ The most frequently cultured bacterial agents found during AECB continue to remain Haemophilus influenzae, Moraxella catarrhalis, and Streptococcus pneumoniae. Others such as enterobacter and Pseudomonas species are found in a minority of cases, $12 \%-16 \%$ and $5 \%-9 \%$, respectively. ${ }^{14}$ The most commonly found atypical organism is Chlamydophila pneumoniae, while Mycoplasma pneumoniae and Legionella pneumophila are rarely isolated. ${ }^{15}$

\section{Use of fluoroquinolones in AECB}

Several recent trials, such as the CAPRIE and MoxiRapid trials, have shown that fluoroquinolones are associated with superior response in AECB. ${ }^{16,17}$ In addition, the Council for Appropriate and Rational Antibiotic Therapy (CARAT) issued a number of guidelines in 2005 to aid physicians in appropriate antibiotic selection when treating AECB in the outpatient setting. They recommended the use of respiratory fluoroquinolones as first-line therapy in the treatment of AECB. The rationale for these recommendations included: the low rates of resistance, broad-spectrum coverage, high respiratory drug concentration and short course therapy. The CARAT criteria also recommend higher-dose, short-course regimens because they improve convenience and increase cost-effectiveness. ${ }^{18}$

\section{The need for new antibiotics}

Prior to the development of the new fluoroquinolones increased resistance in Gram-positive organisms against the older fluoroquinolones lead to increased effort to develop new quinolones with increased activity against these organisms. In Canadian isolates for instance, resistance continues to increase among the entire fluoroquinolone class. Rates of resistance were $1.8 \%, 0.7 \%, 0.6 \%$, and $0.3 \%$ to ciprofloxacin, levofloxacin, gatifloxacin, and moxifloxacin, respectively ${ }^{19}$ in 2002. Overall, the Canadian, TRUST, and PROTEKT US data show that $S$. pnemoniae resistance to flouroquinolones 
is increasing, with current resistance rates ranging between $0.8 \%-1.8 \%{ }^{20}$

Two main strategies have been suggested regarding the appropriate response to the apparent increased resistance rates of $S$. pneumoniae to fluoroquinolones. One strategy limits exposure by restricting fluoroquinolone use to patients who are allergic to $\beta$-lactams, have failed first line therapy, or have infections with resistant organisms ${ }^{21}$ thus avoiding first line use in many patients. The second strategy postulates that using the most potent quinolone as a first-line agent would reduce the risk of treatment failure (because of increased compliance with shorter regimen) and thus reduce the emergence of resistance. ${ }^{22}$

\section{Resistance to fluoroquinolones}

Resistance to fluoroquinolones among Gram-positive bacteria (such as $S$. pneumoniae and $S$. aureus) (23 $^{23}$ occurs by two mechanisms. Alterations in the genes coding for both subunits of DNA gyrase ( $g y r A$ and $g y r B$ ) or topoisomerase $\operatorname{IV}(\operatorname{par} C$ and $\operatorname{par} E)$ is the first mechanism and the second is through the activity of efflux pumps. The gyrase and topoisomerase mutations may coexist. However, parC mutations always precede gyrA mutations in S. pneumoniae. Fluoroquinolone concentrations that are effective in preventing the first mutation ( $\mathrm{parC}$ ) will decrease the development of resistance since muations in gyrA do not usually appear in the absence of that first step. ${ }^{24}$

Fluoroquinolone resistance develops by stepwise selection of mutations in a region that encodes for the above enzymes, also known as the quinolone resistance-determining region (QRDR). Target preference varies between different fluoroquinolones. Ciprofloxacin and levofloxacin, for instance, target topoisomerase IV primarily. Moxifloxacin, on the other hand targets DNA gyrase. ${ }^{25}$ In contrast, gemifloxacin has high affinity for pneumococcal topoisomerase IV and an ability to inhibit both DNA gyrase and topoisomerase IV. ${ }^{26}$

\section{Gemifloxacin}

Approved by the US Food and Drug Administration (FDA) in April 2003, gemifloxacin is a member of the fluoroquinolone class that was originally introduced in the 1980s. Typified by ciprofloxacin quinolones had good broad spectrum activity, including activity against $P$ seudomonas aeruginosa, user-friendly pharmacokinetics allowing twice-daily dosing, as well as a benign side-effect profile. Ciprofloxacin's activity against $S$. pneumoniae was its main shortcoming, with a minimal inhibitory concentration (MIC) close to susceptibility breakpoints. Several respiratory fluoroquinolones were later introduced with modifications meant to increase activity against Gram-positive pathogens. ${ }^{27}$ Several such new agents as grepafloxacin, sparfloxacin, trovafloxacin, and gatifloxacin had to be withdrawn from the market due to toxicities, but levofloxacin, moxifloxacin, and gemifloxacin remain the respiratory fluoroquinolones currently available in the United States.

\section{Chemistry}

The basic structure for the development of the newer quinolones continues to be the nalidixic acid base, with certain structural modifications to the aromatic core. All the new fluoroquinolones retain the 3-carboxyl and ketone groups at C-4 from the basic quinolone structure, both of which have a role in the binding to the DNA-gyrase complex. Gemifloxacin in particular has a nitrogen in place of the carbon at C-8 change which is thought to enhance its activity against DNA gyrase and topoisomerase IV (Figure 1). ${ }^{28}$

\section{Pharmacokinetics}

The new fluoroquinolones, and by extension gemifloxacin, are absorbed rapidly after oral administration with maximum plasma concentration $\left(\mathrm{C}_{\max }\right)$ obtained in 1-3 hours. A single $320 \mathrm{mg}$ dose of gemifloxacin yields a peak plasma concentration of $1.6 \mathrm{mg} / \mathrm{L}$ after one hour. Administration with food may delay absorption, however studies by Russo and colleagues and Gajjar and colleagues showed that overall bioavailability of garenoxacin (des-F(6)-quinolone BMS-284756) are equivalent in the fed and fasting states. ${ }^{29,30}$ indicating that perhaps fluoroquinolone bioavailability and pharmacokinetic properties may not be significantly affected by food intake or high fat meals (Table 2).

Penetration of the new flouroquinolones is excellent, particularly into alveolar macrophages, bronchial mucosa, epithelial lining fluid, and saliva, even though penetration into the cerebrospinal fluid (CSF) is limited. Gemifloxacin shows similar penetration as other quinolones into saliva, CSF, and

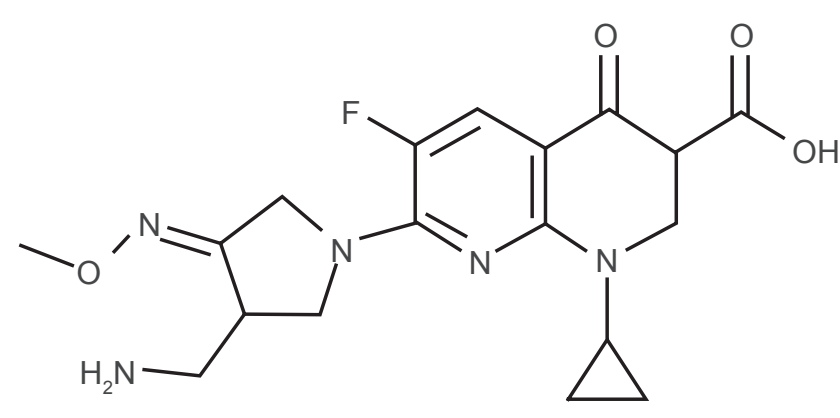

Figure I Chemical structure of gemifloxacin. 
Table I Comparison of in vitro activity of gemifloxacin and other quinolones against the typical ABECB organisms. Adapted from Zhanel GG, Fontaine S, Adam H, et al. A review of new fluoroquinolones - focus on their use in respiratory tract infections. Treat Respir Med. 2006;5(6):437-465. ${ }^{31}$ Copyright (C) 2006, with permission from Wolters Kluwer Health pharma Solutions

\begin{tabular}{|c|c|c|c|c|c|c|}
\hline \multirow[t]{2}{*}{ Antibiotic } & \multicolumn{2}{|c|}{ Streptococcus pneumoniae } & \multicolumn{2}{|c|}{ Haemophilus inluenzae } & \multicolumn{2}{|c|}{ Moraxella catarrhalis } \\
\hline & $\mathrm{MIC}_{50}$ & $\mathrm{MIC}_{90}$ & $\mathrm{MIC}_{50}$ & $\mathrm{MIC}_{90}$ & $\mathrm{MIC}_{50}$ & $\mathrm{MIC}_{90}$ \\
\hline Gemifloxacin & 0.03 & 0.03 & 0.004 & 0.008 & 0.015 & 0.015 \\
\hline Levofloxacin & I & I & 0.015 & 0.03 & 0.06 & 0.06 \\
\hline Ciprofloxacin & I & 2 & 0.004 & 0.008 & 0.03 & 0.03 \\
\hline Moxifloxacin & 0.12 & 0.12 & 0.03 & 0.03 & 0.06 & 0.06 \\
\hline
\end{tabular}

Abbreviations: $\mathrm{MIC}_{50 / 90}$, drug concentration inibiting $50 \%$ and $90 \%$ of the number of strains tested in ug/ml.

inflammatory blister fluid. ${ }^{31}$ Of note is the comparatively low concentration gemifloxacin has in the serum when compared to levofloxacin or moxifloxacin $(1.4 \mathrm{mg} / \mathrm{L}$ vs $4.9 \mathrm{mg} / \mathrm{L}$ and $3.2 \mathrm{mg} /$ $\mathrm{L}$, respectively). Despite this, gemifloxacin has achieved bronchial mucosa levels and alveolar macrophage levels higher than the other two quinolones. Gemifloxacin achieves higher drug levels in respiratory tissues than in serum. ${ }^{27}$

The new fluoroquinolones in addition have a longer halflife than ciprofloxacin, and all are at least partially eliminated through renal pathways. Gemifloxacin and moxifloxacin are mostly eliminated by nonrenal pathways. However, dosage adjustment for gemifloxacin for renally impaired patients is still required. ${ }^{31}$

\section{Pharmacodynamics}

The free 24-hour area under the serum drug concentration/time curve (AUC) to MIC ratio is thought to be the major pharmacodynamic predictor of fluoroquinolone efficacy. ${ }^{27}$ However, the $\mathrm{C}_{\max } / \mathrm{MIC}$ ratio may also be predictive, since in human studies regarding the activity of levofloxacin the $\mathrm{C}_{\max } / \mathrm{MIC}$ ratio predicted clinical outcome and microbiologic eradication better than AUC/MIC. ${ }^{34}$ Even clinical trials that showed AUC/MIC as the most important parameter, showed that a high $\mathrm{C}_{\max } / \mathrm{MIC}$ may prevent selection against resistant bacterial strains. ${ }^{35}$ For all quinolones the target $\mathrm{AUC}_{24} / \mathrm{MIC}$ to be achieved for Gram-positive coverage in immunocompetent patients is $\geq 25-30$, and in immunocompromised patients $\geq 100-125 .{ }^{27}$ Like other fluoroquinolones, gemifloxacin is considered to be a concentration-dependent bacterial killer and its activity is predicted by its ability to reach the target $\mathrm{AUC}_{24} / \mathrm{MIC}$. If we apply this ratio to evaluate activity against $S$. pneumoniae we find that gemifloxacin achieved a target of 97-127. By comparison moxifloxacin achieved a ratio of 96 , while ciprofloxacin did not reach a ratio of $25 .^{36}$

Other pharmacodynamic parameters used more recently are the postantibiotic effect (PAE) and the mutant prevention concentration (MPC). PAE measures the continued suppression of bacterial growth after exposure, which means that prolonged PAEs protect against bacterial regrowth during medication troughs. Given that the PAE of fuoroquinolones is between 1.5-2.5 hours this permits the 12 hour or 24 hour dosing of fluoroquinolones. ${ }^{29}$ Gemifloxacin has been shown to have significant in vitro PAE against strains of S. pneumoniae and H. influenzae. ${ }^{38,39}$ The MPC is the drug concentration at which selection for resistance is inhibited and many of the newer fluoroquinolones can be administered safely at concentrations that reach the MPC. ${ }^{40}$ Gemifloxacin was found to have an MPC of $0.13 \mu \mathrm{g} / \mathrm{ml}$ (the lowest of all the new fluoroquinolones) against $S$. pnemunoiae $e^{31,32}$ and $0.125 \mu \mathrm{g} / \mathrm{ml}$ against $H$. influenzae. ${ }^{33}$

Table 2 Summary of the clinical and bacterial efficacy of gemifloxacin versus other agents in the treatment of AECB. Adapted from Appelbaum PC, Gillespie SH, Burley CJ, Tillotson GS. Antimicrobial selection for community-acquired lower respiratory tract infections in the 2 I st century: a review of gemifloxacin. Int J Antimicrob Agents. 2004;23:533-546. Copyright @ 2004, with permission from Elsevier

\begin{tabular}{|c|c|c|c|c|c|}
\hline \multirow[t]{2}{*}{ Study } & \multirow[t]{2}{*}{ Comparator } & \multicolumn{2}{|c|}{ Clinical success in PPP \% } & \multicolumn{2}{|c|}{ Bacteriologic success in PPP \% } \\
\hline & & Gemifloxacin & Comparator & Gemifloxacin & Comparator \\
\hline Ball et $\mathrm{al}^{52}$ & None & 89.6 & & 91.7 & \\
\hline Ball et al ${ }^{6}$ & Trovafloxacin & 91.5 & 87.6 & 86.8 & 82.4 \\
\hline Wilson et al ${ }^{49}$ & Ceftriaxone/cefuroxime & 86.8 & 81.3 & $62.5^{\mathrm{a}}$ & $60.8^{\mathrm{a}}$ \\
\hline Wilson et al $\left.\right|^{51}$ & Clarithromycin & 85.4 & 84.6 & 86.7 & 73.1 \\
\hline File et $\mathrm{al}^{51}$ & Amoxicillin/clavulanate & 93.6 & 93.3 & 90 & 79.5 \\
\hline Sethi et $\mathrm{a}^{54}$ & Levofloxacin & 88.2 & 85.1 & & \\
\hline
\end{tabular}

Note: a - ITT population

Abbreviation: PPP per-protocol population 
In their assessment of pharmacokinetic-pharmacodynamic (PK-PD) target attainment of gemifloxacin against S. pneumoniae in a simulated population of 2500 patients in 2005, Owens and colleagues analyzed the probability of attaining fAUC/MIC (24-hour free AUC/MIC) ratio based on data from two sources. ${ }^{41}$ First Owens used a population pharmacokinetic model and second, an MIC distribution based on 3317 S. pneumoniae isolates was used. The probability of attaining fAUC/MIC ratio was 0.79 for levofloxacin and 0.98 for gatifloxacin. In another analysis the probability was $85.8 \%$ for levofloxacin and $99.3 \%$ for moxifloxacin. The probability of reaching the PK-PD target for gemifloxacin "over the entire pneumococcal MIC distribution was greater than 0.99." Owens and collegues concluded that in regions where increased incidence of levofloxacin-resistant pneumococci has been recorded, such as the western United States, it might be prudent to use more potent agents like gemifloxacin instead of the less potent levofloxacin since gemifloxacin has a higher probability of reaching the intended pharmacodynamic and pharmacokinetic targets.

\section{In vitro activity}

A number of in vitro studies done over the last few years compared the activity of new and old fluroquinolones against the typical respiratory organisms associated with AECB. Table 1 contains the results of some several in vitro studies as well as the $\mathrm{MIC}_{50}$ and $\mathrm{MIC}_{90}$ for ciprofloxacin, levofloxacin, moxifloxacin, and gemifloxacin. In all cases gemifloxacin has a lower MIC than the other fluoroquinolones.

In a 2004 study, Pereyre and colleagues compared garenoxacin (a des-fluoro quinolone unavailable in the United States), gatifloxacin and gemifloxacin with four other quinolones: moxifloxacin, levofloxacin, ciprofloxacin, and ofloxacin..$^{42}$ These antibiotics were tested against 31 strains of Mycoplasma pneumonieae, 40 strains of M. hominis, 46 strains of Ureaplasma and 9 strains of M. fermentans. Of the new fluoroquinolones garenoxacin and gemifloxacin had the highest activity against quinolone-resistant strains of both Mycoplasma and Ureaplasma with MICs of $\leq 1 \mu \mathrm{g} / \mathrm{ml}$ for all strains with one mutation and most of the strains with two mutations.

In 2005, Pankuch and colleagues tested the activity of five quinolones (ciprofloxacin, levofloxacin, gatifloxacin, moxifloxacin, and gemifloxacin), three macrolides (erythromycin, azithromycin, and clarithromycin), and telithromycin against $12 \mathrm{H}$. influenzae strains. ${ }^{43}$ All quinolones were active against all 12 strains tested regardless of the $\beta$-lactam or macrolide resistance.
In 2005, an in vitro study in Saudi Arabia tested ciprofloxacin, ofloxacin, gemifloxacin, grepafloxacin, trovafloxacin, and levofloxacin against the most common bacteria associated with AECB. ${ }^{44}$ Eighty-eight isolates of $S$. pneumoniae, 116 strains of $H$. influenzae, and 80 strains of $M$. catarrhalis were used in the comparison. The authors found that $S$. pneumoniae isolates were fully susceptible to trovafloxacin, grepafloxacin, and gemifloxacin while susceptibility to ofloxacin and levofloxacin was $97.7 \%$ and 98.9\%. H. influenzae was susceptible to all agents except trovafloxacin (99.1\%) and M. catarrhalis was susceptible to all agents except ofloxacin (97.5\%). None of these isolates were resistant to gemifloxacin.

In 2006, De Azavedo and colleagues tested serotype 6B of the BCP2443 strain of $S$. pneumoniae against gemifloxacin, gatifloxacin, and goxifloxacin. ${ }^{45}$ This isolate is resistant to erythromycin and intermittently resistant to penicillin, but fully susceptible to all fluoroquinolones and has no known quinolone resistance-determining region (QRDR) mutations. They found that gemifloxacin showed 2- to 16-fold greater activity than moxifloxacin or gatifloxacin against strains with two or more QRDR mutations.

Finally, in 2007, La Plante and colleagues analyzed the association between fAUC/MIC ratio and the development of resistance of wildtype $S$. pneumoniae to gatifloxacin, gemifloxacin, levofloxacin, and moxifloxacin. ${ }^{46}$ They found that gatifloxacin, gemifloxacin, and moxifloxacin exceeded the fAUC/MIC resistance breakpoint against $S$. pneumoniae. They also found that the order of resistance development determined from fAUC/MIC breakpoints was levofloxacin $>$ gatifloxacin $>$ moxifloxacin $=$ gemifloxacin. These results show that moxifloxacin and gemifloxacin have optimal in vitro activity against wildtype $S$. pnumoniae, and suggest that their use may decrease the emergence of resistance in S. pneumoniae.

\section{Animal studies}

Animal studies done to compare gemifloxacin with other respiratory antibiotics and other fluoroquinolones continue the same trend seen in the in vitro results, showing better clinical response after treatment with gemifloxacin versus some of the older and newer fluoroquinolones, as well as versus other antibiotics commonly used against respiratory pathogens.

Berry and colleagues tested gemifloxacin in the rat respiratory tract infection against four strains of $S$. pneumoniae and two strains of $H$. influenzae and compared it with amoxicillin-clavulanate, ciprofloxacin, cefuroxime, azithro- 
mycin, trovafloxacin, grepafloxacin, and levofloxacin. ${ }^{47}$ Their results showed that gemifloxacin was as effective as amoxicillin-clavulanate, and more potent than all others against the strains of $S$. pneumoniae. Gemifloxacin was significantly more potent than cefuroxime and azithromycin against the H. influenzae strains.

In the Swiss White mouse model Bast and colleagues compared two-day and five-day courses of gemifloxacin versus levofloxacin in the treatment of pneumococcal pneumonia. ${ }^{48}$ Survival rates for gemifloxacin were $83 \%-100 \%$ compared with 40\%-58\% for levofloxacin.

\section{Clinical studies}

Several noninferiority clinical trials comparing gemifloxacin to other antibiotics used in the treatment of AECB have been completed since gemifloxacin's introduction in 2003, however so far only two compared gemifloxacin with other fluoroquinolones. Although these noninferiority trials were not designed to highlight the difference between gemifloxacin and comparator antibiotics, a few differences should be noted.

In our review we found three studies that compared gemifloxacin with ceftriaxone/cefuroxime, clarithromycin and amoxicillin/clavulanate respectively. In 2003, Wilson and colleagues compared the activity of gemifloxacin with that of IV ceftriaxone/oral cefuroxime in the treatment of 271 hospitalized patients with AECB. ${ }^{49}$ The clinical success rates at follow-up were $86.8 \%$ for gemifloxacin vs. $81.3 \%$ for ceftriaxone/cefuroxime in the entire study population (treatment difference $=5.5 ; 95 \%$ confidence interval $[\mathrm{CI}]:-3.9,14.9)$. In the clinical intention-to-treat (ITT) population the clinical results were $82.6 \%$ vs. $72.1 \%$ (95\% CI: 0.7, 20.4). Time to discharge for the gemifloxacin group was nince days while for the IV/oral $\beta$-lactam group was 11 days. This decrease in time of hospitalization in the gemifloxacin group was statistically significant $(p=0.04$.)

In another British noninferiority trial, 712 patients with Anthonisen type I AECB symptoms (increased dyspnea, cough, and sputum purulence) were randomized to treatment with five days of gemifloxacin $(n=351)$ versus seven days of clarithromcin $(n=361) .{ }^{50}$ The long-term phase of the study (26 weeks) evaluated the percentage of patients who remained free of $\mathrm{AECB}$ and did not require reinitiation of antibiotic treatment. Clinical success rates were $85.4 \%$ for gemifloxacin and $84.6 \%$ for clarithromycin at the 2-3 week follow-up. Bacteriologic success rates were $86.7 \%$ for gemifloxacin versus $73.1 \%$ for clarithromycin. In addition, in the gemifloxacin group $71 \%$ of patients remained free of AECB symptoms at the 26-week follow-up, versus $58.5 \%$ for clarithromycin $(\mathrm{p}=0.016)$. Of note in this study is that a significantly shorter time to $H$. influenzae eradication was noted in the gemifloxacin group versus the clarythromycin group $(\mathrm{p}=0.02)$ : no $H$. influenzae was recovered from the gemifloxacin group after one day of treatment, while $50 \%$ were recovered from the clarithromycin group.

In a 2000 comparison study done in the United States, File and colleagues compared gemifloxacin with amoxicillin/clavulanate for the treatment of AECB. ${ }^{51}$ The clinical success rates were comparable at $93.6 \%$ for gemifloxacin versus $93.2 \%$ for amoxicillin/clavulanate. The bacteriological success rate of gemifloxacin however was significantly higher: $90.0 \%$ versus $79.5 \%$ difference which achieves statistical significance (95\% CI: -3.3 to 26.0 ).

In the two studies that compared gemifloxacin with another fluoroquinolone, gemifloxacin showed higher clinical success rates versus trovafloxacin and levofloxacin respectively. In 2001, Ball and colleagues published a prospective, randomized, double-blind, double dummy, parallel, multicenter comparison of gemifloxacin with trovafloxacin in 617 randomized patients with AECB. ${ }^{52}$ The clinical success rates were $91.5 \%$ for gemifloxacin versus $87.6 \%$ for trovafloxacin. In the ITT population, clinical success was $89 \%$ for gemifloxacin versus $83 \%$ for trovafloxacin, which was statistically significant (95\% CI: $-1.2,9.0)$. Clinical recurrence rates at long-term follow-up were $8.8 \%$ for gemifloxacin versus $10.5 \%$ for trovafloxacin.

In a second study, this time an open-label, noncomparative study, Ball and colleagues assessed the clinical efficacy of gemifloxacin in AECB and community-acquired pneumonia (CAP) and reported clinical success of $83.1 \%$ at follow-up and bacteriologic success (ITT) of 91.2\%. ${ }^{53}$ Total eradication rates exceeded $90 \%$ in this study including eradication of $H$. influenzae, M. catarrhalis, and S. pneumoniae (S. pneumoniae was eradicated in all cases).

Finally in 2004, Sethi and colleagues compared the clinical efficacy of gemifloxacin versus levofloxacin in 360 patients with AECB in their randomized, doubleblind, double dummy, multicenter, parallel group study. ${ }^{54}$ Clinical success rate in the protocol population was $88.2 \%$ for gemifloxacin and $85.1 \%$ for levofloxacin. In the ITT population the clinical success rate was $85.2 \%$ for gemifloxacin versus $78.1 \%$ for levofloxacin. At long-term follow-up (days 28-35) the gemifloxacin success rate was $83.7 \%$ versus $78.4 \%$ for levofloxacin, which is a clinically significant finding (95\% CI: $-3.83,14.34)$, and fewer patients withdrew from the gemifloxacin group than the levofloxacin group 
(seven patients vs 18 patients, respectively, which was also statistically significant; $\mathrm{p}=0.02$ ).

These clinical trials, although intended to show the noninferiority of gemifloxacin, identified some clinically significant differences between gemifloxacin and comparator drugs: decreased length of hospitalization versus ceftriaxone/cefuroxime; ${ }^{49}$ shorter time to eradication of $H$. influenzae when compared with clarithromycin; $; 0$ increased bacteriological success rate in AECB when compared to amoxicillin/clavulanate. ${ }^{51}$ More importantly for our purposes of comparing gemifloxacin with other fluoroquinolones, gemifloxacin had a higher clinical success rate when compared to trovafloxacin, ${ }^{52}$ and a higher rate of success versus levofloxacin at long-term follow-up ${ }^{54}$ in two high quality trials (both trials get high scores on Jadad scale). ${ }^{55}$

\section{Clinical tolerability}

The most common adverse reactions to fluoroquinolones are mild and consist of gastrointestinal (GI) symptoms, central nervous system (CNS) symptoms, and skin symptoms. ${ }^{31}$ Gastrointestinal reactions include nausea, vomiting, and diarrhea. Nausea is the most common GI symptom and is more common with gatifloxacin and moxifloxacin ranging between $6 \%-10 \%$, while vomiting and diarrhea range between $1 \%-6 \%$ and appear to be similar among the newer fluoroquinolones. CNS adverse reactions have been reported with all fluoroquinolones and consist mainly of headaches and dizziness. These reactions have been reported to be $2 \%-3 \%$ for gatifloxacin and moxifloxacin versus $1 \%$ for gemifloxacin and levofloxacin.

Gemifloxacin has been associated with 2.8\%-4.8\% incidence of hypersensitivity reactions (rash and pruritus), which is the highest rate of all the new fluoroquinolones as opposed to $1 \%$ for the others. These reactions are most common in women aged younger than 40 years as well as in postmenopausal women taking estrogen replacement. ${ }^{31}$ The rash was also associated with length of duration of therapy, usually noticed on regimen longer than seven days. ${ }^{27}$

Photosensitivity has also been reported with several fluoroquinolones and the associated skin changes consist of erythema, edema, desquamation and rarely painful blistering. In the case of gemifloxacin, phototoxicity appears to be dose-related and resolves within 48 hours after treatment is stopped. Photoxocity has not been reported with moxifloxacin or gatifloxacin.

QT prolongation is a well-reported adverse reaction to fluoroquinolones. A retrospective database analysis for the period 1996-2001 in the USA has found 25 cases of torsades de pointes (Tdp) ${ }^{56}$ associated with use of fluoroquinolones. However, the proarrhythmic potential is not the same for all fluoroquinolones. Moxifloxacin seems to have the greatest risk of QT prolongation, while gemifloxacin, ciprofloxacin, and ofloxacin seem to have less risk. The overall risk of developing Tdp is low at less than that of macrolides ${ }^{57,58}$ however, current recommendations still advise caution in prescribing fluoroquinolones to patients who are taking other medications that may prolong the QT interval. Electrocardiogram monitoring is not necessary in all patients, but should be done in patients who have underlying conditions that may cause QT prolongation or who take other pharmaceuticals that may cause it. ${ }^{59}$

Hepatotoxicity, characterized by increased aspartate aminotransferase and alanine aminotransferase $>1.5$ times baseline has been reported in $4.1 \%$ of patiens on gemifloxacin. Moxifloxacin was associated with $2.5 \%-3.8 \%$ hepatotoxicity. ${ }^{31}$

Several cases of arthralgias have been reported in association with fluoroquinolones in children, but there is no evidence of decreased linear growth in treated children. Most of the children treated with respiratory fluoroquinolones have cystic fibrosis. The rate of treatment related arthralgia was no greater than the rate in nontreated cystic fibrosis patients. ${ }^{31}$

Tendinopathy is another rare but well-documented class effect with fluoroquinolones. In $90 \%$ of the reported cases of tendinopathy, the Achilles tendon was the injured tendon. Risk factors include concurrent therapy with corticosteroids or hemodialysis, or the presence of renal dysfuction, post-renal transplant, and rheumatic diseases. ${ }^{31}$ A recent boxed warning about tendinopathy was added to all fluoroquinolone package inserts. ${ }^{60}$

\section{Economics}

A cost analysis of the GLOBE study data evaluated the cost effectiveness of gemifloxacin versus clarithromycin in the treatment of AECB. In their analysis, Halpern and colleagues found that of the patients treated with gemifloxacin $73.8 \%$ did not have recurrence of AECB versus $63.8 \%$ of the clarithromycin group $(\mathrm{p}=0.024) .{ }^{61}$ Fewer patients on gemifloxacin therapy were hospitalized than patients on clarithromycin therapy $(2.33 \%$ versus $6.25 \%$; $=0.059)$. The mean direct cost for a patient on gemifloxacin was US\$247 versus US\$347 for a patient on clarithromycin. This represents a $29 \%$ difference in mean direct cost. The mean direct and indirect costs for a patient on gemifloxacin was US $\$ 1,413$ versus US\$1,742 for a patient on clarithromycin, or a $19 \%$ difference in indirect costs. 
In 2003, Wilson and colleagues showed that the time to hospital discharge for the gemifloxacin group was nine days, while the time for the IV/oral $\beta$-lactam group was 11 days $(\mathrm{p}=0.04$. $) .{ }^{50} \mathrm{In}$ addition, clinical success rates at follow-up (21-28 days post therapy) of $86.8 \%$ for gemifloxacin versus $81.3 \%$ for ceftriaxone/cefuroxime (95\% CI: $-3.9,14.9$ ) indicate a possible lower frequency of treatment failure. These findings suggest that gemifloxacin can result in cost savings when compared to the comparators ceftriaxone/oral cefuroxime, and clarithromycin.

\section{Conclusion}

Gemifloxacin is a new fluoroquinolone with proven efficacy against respiratory bacteria associated with AECB. In vitro and clinical studies show that gemifloxacin is not inferior to the other respiratory fluoroquinolones, and may have some potential advantages. It has a lower MIC than other new fluoroquinolones against the typical pathogens in AECB; it has dual activity against DNA gyrase and topoisomerase IV, and it has been associated with decreased emergence of resistance in S. pneumoniae. Clinically it has been associated with decreased length of hospitalization when compared to ceftriaxone and clarithromycin (and as a result with decreased health care costs) and higher rate of success versus levofloxacin at longterm follow-up. Gemifloxacin has a slightly higher incidence of hepatotoxicity and hypersensitivity reactions than other new fluoroquinolones, while it appears to have a lower incidence of gastrointestinal and CNS symptoms. In conclusion, gemifloxacin seems to be an effective quinolone in the treatment of AECB, however further studies comparing gemifloxacin with other respiratory quinolones are needed to determine any possible superiority. Its long-term benefit could be based on a theoretic ability to decrease emergence of quinolone resistance in respiratory pathogens. A well characterized rash on top of other known quinolone side effects can occur.

\section{Disclosure}

Dr Jivcu does not have any conflict of interest or disclosures in this work. Dr Gotfried has received research support by Johnson and Johnson and he was a consultant for Schering-Plough.

\section{References}

1. Global Initiative for Chronic Obstructive Lung Disease. Global Strategy for Diagnosis, Management, and Prevention of COPD. 2007. Accessed on January 10, 2009. Available from: http://www.goldcopd. com/download.asp?intId=502. p. 15.

2. American Thoracic Society. Standards for the diagnosis and care of patients with chronic obstructive pulmonary disease. Am J Respir Crit Care Med. 1995:152:S77-S121.
3. Wedzicha JA. Exacerbations: etiology and pathophysiologic mechanisms. Chest. 2002;12(Suppl 5):136S-141S.

4. Van Weel C. Chronic diseases in general practice: the longitudinal dimension. Eur J Gen Pract. 1996;2:17-21.

5. Murphy TF, Sethi S. Bacterial infection in chronic obstructive pulmonary disease. Am Rev Respir Dis. 1992;146:1067-1083.

6. Wilson R. A vicious circle hypothesis operating during infective exacerbations of chronic bronchitis. Monaldi Arch Chest Dis. 1994;49(2):159-164.

7. Sethi S, Murphy TF. Acute exacerbations of chronic bronchitis: new developments concerning microbiology and pathophysiology - impact on approaches to risk stratification and therapy. Infect Dis Clin North Am. 2004;18:861-882.

8. Martinez FJ, Curtis JL. Procalcitonin-guided antibiotic therapy in COPD exacerbations: closer but not quite there. Chest. 2007;131(1):9-19.

9. Anthonisen NR, Manfreda J, Warren CPW, Hershfield ES, Harding GKM, Nelson NA. Antibiotic therapy in exacerbations of chronic obstructive pulmonary disease. Ann Intern Med. 1987;106:196-204.

10. Balter MS, La Forge J, Low DE, et al. Canadian guidelines for the management of acute exacerbations of chronic bronchitis: executive summary. Can Respir J. 2003;10:248-258.

11. Stephen B, Blaine PC, Richard C, et al. Acute exacerbation of chronic bronchitis: A primary care consensus guideline. Am J Manage Care. 2004;10:689-696.

12. Flaherty KR, Sain S, Fendrick AM, Martinez FJ. The spectrum of acute bronchitis: using baseline factors to guide empirical therapy. Postgrad Med. 2001;109:39-47.

13. Sethi S. Infectious exacerbations of chronic bronchitis: diagnosis and management. J Antimicrob Chemother. 1999;43(Suppl A):97-10.

14. Kahn JB, Khashab M, Ambruzs M. Study entry microbiology in patients with acute bacterial exacerbation of chronic bronchitis in a clinical trial stratifying by disease severity. Curr Med Res Opin. 2007;23(1):1-7.

15. Sethi S. Infectious etiology of acute exacerbations of chronic bronchitis. Chest. 2000;117(5 Suppl 2):380S-3805S.

16. Anzueto A, Niederman MS, Pearle J, Restrepo MI, Heyder A, Choudhri SH; Community-Acquired Pneumonia Recovery in the Elderly Study Group. Community-Acquired Pneumonia Recovery in the Elderly (CAPRIE): efficacy and safety of moxifloxacin therapy versus that of levofloxacin therapy. Clin Infect Dis. 2006;42:73-81.

17. Welte T, Petermann W, Schürmann D, Bauer TT, Reimnitz P; MOXIRAPID Study Group. Treatment with sequential intravenous or oral moxifloxacin was associated with faster clinical improvement than was standard therapy for hospitalized patients with community-acquired pneumonia who received initial parenteral therapy. Clin Infect Dis. 2005;41(12):1697-705.

18. Fernando JM, Anzueto A. Appropriate outpatient treatment of acute bacterial exacerbation of chronic bronchitis. Am J Med. 2005; 118(Suppl 7A):39S-44S.

19. Tang P, Green K, McGeer A, et al. Fluoroquinolone resistance in Streptococcus pneumoniae in Canada - are we currently surveying just the tip of the iceberg? In: Program and abstracts of the 42nd Interscience Conference on Antimicrobial Agents and Chemotherapy (San Diego). Washington, DC: American Society for Microbiology; 2002. p. 110.

20. Kelly L. Multidrug-resistant pneumococci isolated in the US: 1997-2001 TRUST surveillance [abstract]. In: Program and abstracts of the 41st Interscience Conference on Antimicrobial Agents and Chemotherapy (Chicago). Washington, DC: American Society for Microbiology; 2001. p. 142.

21. Niederman MS. Guidelines for the management of community-acquired pneumonia. Current recommendations and antibiotic selection issues. Med Clin North Am. 2001:85:1493-1509.

22. Doern GV. Antimicrobial use and the emergence of antimicrobial resistance with Streptococcus pneumoniae in the United States. Clin Infect Dis. 2001;33(Suppl 3):S187-S192.

23. Kaatz GW, Seo SM. Mechanism of fluoroquinolone resistance in genetically related strains of Staphylococcus aureus. Antimicrob Agents Chemother. 1997;12:2733-2737. 
24. LaPlante KL, Rybak MJ, Rsuji B, Lodise TP, Kaatz GW. Fluoroquinolone resistance in Streptococcus pneumoniae: area under the concentrationtime curve/MIC ratio and resistance development with gatifloxacin, gemifloxacin, levofloxacin, and moxifloxacin. Antimicrob Agents Chemother. 2007;51(4):1315-1320.

25. Smith HJ, Hoban DJ, Zhanel GG. Dual activity of fluoroquinolones against Streptococcus pneumoniae: the facts behind the claims. J Antimicrob Chemorther. 2002;49:893-895.

26. De Azavedo JCS, Duncan CL, Kilurn L, et al. Relative potential for selection of quinolone-resistance-determining-region mutations in Streptococcus pneumoniae by gemifloxacin, gatifloxacin and moxifloxacin. J Chemother. 2006;18(4):373-378.

27. Appelbaum PC, Gillespie SH, Burley CJ, Tillotson GS. Antimicrobial selection for community-acquired lower respiratory tract infections in the 21st century: a review of gemifloxacin. Int $J$ Antimicrob Agents 2004;23:533-546.

28. Yoo BK, Triller DM, Yong CS, Lodise TP. Gemifloxacin: a new fluoroquinolone approved for treatment of respiratory infections Ann Pharmacother. 2004;38(7-8):1226-1235.

29. Russo R, Bello JA, Christopher L, et al. Lack of effect of high-fat meal on the bioavailability of garenoxacin oral suspension in healthy subjects. Chicago, IL: American Society of Hospital Pharmacists (ASHP) Midyear Clinical Meeting; 2002.

30. Gajjar DA, Sukoneck SC, Bello A, Ge Z, Christopher L, Grasela DM. Effect of a high-fat meal on the pharmacokinetics of the des-F(6)-quinolone BMS-284756. Pharmacotherapy. 2002;22:160-165.

31. Zhanel GG, Fontaine S, Adam $\mathrm{H}$, et al. A review of new fluoroquinolones focus on their use in respiratory tract infections. Treat Respir Med. 2006;5(6): $437-465$

32. Smith HJ, Hoban DJ, Zhanel GG, et al. Molecular characterization and mutatnt prevention concentration (MPC) determination of single-step fluoroquinolone resistant mutants of S.pneumoniae. Chicago, IL: 41st Interscience Conference of Antimicrobial Agents and Chemotherapy; 2001:22-25.

33. Blondeau JM, Borsos S. Comparative minimum inhibitory concentration and mutant preveniton concentration of azithromycin, cefuroxime, gemifloxacin, moxifloxacin and telithromycin against clinical isolates of Haemophilus influenzae. Munich, Germany: 17th European Congress of Clinical Microbiology and Infectious Diseases (ECCMID); 2007 March 31-April 3. Abstract P-747.

34. Zhanel GG, Ennnis K, Vercaigne L, et al. A critical review of the fluoroquinolones: focus on respiratory infections. Drugs. 2002;62(1):13-59.

35. Tran JQ, Ballow CH, Forrest A, et al. Comparison of the abilities of grepafloxacin and clarithromycin to eradicate potential bacterial pathogens from the sputa of patients with chronic bronchitis: influence of pharmacokinetic and pharmacodynamic variables. J Antimicrob Chemother. 2000;45:9-17.

36. King A, May J, Grench G, Phillips I. Comparative in vitro activity of gemifloxacin. J Antimicrob Agents. 2003;21:574-577.

37. Thomas KS, Forrest A, Bhavnani SM, et al. Pharmacodynamic evaluation of factors associated with the development of bacterial resistance in acutely ill patients during therapy. Antimicrob Agents Chemother. 1998;42:521-527.

38. Davies TA, Kelly LM, Pankush GA, Credito KL, Jacobs MR, Appelbaum PC. Antipneumococcal activities of gemifloxacin compared to those of nine other agents. Antimicrob Agents Chemother. 2002;46:267-274.

39. Davies TA, Kelly LM, Hoellman DB, et al. Activities and postantibiotic effects of gemifloxacin compared to those of 11 other agents against Haemophilus influenzae and Moraxella catarrhalis. Antimicrob Agents Chemother. 2000;44:633-639.

40. Drlica K. A strategy for fighting antibiotic resistance. ASM News. 2001;67:27-33.

41. Owens RD, Bhavnani SM, Ambrose PG. Assessment of pharmacokineticpharmacodynamic target attainment of gemifloxacin against Streptococcus pneumoniae. Diagn Microbiol Infect Dis. 2005;51:45-49.
42. Pereyre S, Renaudin H, Bébéar C, Bébéar CM. In vitro activities of the newer quinolones garenoxacin, gatifloxacin, and gemifloxacin against human mycoplasmas. Antimicrob Agents Chemother. 2004;48(8):3165-3168.

43. Pankuch GA, Lin G, Appelbaum PC. Activity of five quinolones, three macrolides and telithromycin against 12 Haemophilus influenzae strains with different resistance phenotypes. Clin Microbiol Infect. 2005;11(12):1040-1044.

44. Balkhy $\mathrm{HH}$, Memish ZA, Shibi A, et al. In vitro activity of quinolones against $S$. pneumoniae, H. influenzae and M. catarrhalis in Saudi Arabia. East Mediterr Health J. 2005;11(1-2):36-44.

45. De Azavedo JCS, Duncan CL, Kilurn L, et al. Relative potential for selection of quinolone-resistance-determining-region mutations in Streptococcus pneumoniae by gemifloxacin, gatifloxacin and moxifloxacin. J Chemother. 2006;18(4):373-378.

46. LaPlante KL, Rybak MJ, Tsuji B, Lodise TP, Kaatz GW. Fluoroquinolone resistance in Streptococcus pneumoniae: area under the concentration-time curve/MIC ratio and resistance development with gatifloxacin, gemifloxacin, levofloxacin, and moxifloxacin. Antimicrob Agents Chemother. 2007;51(4):1315-1320.

47. Berry V, Page R, Satterfield J, Singley C, Straub R, Woodnutt G. Comparative in vivo acitivity of gemifloxacin in a rat model of respiratory tract infection. J Antimicrob Chemother. 2000;45(Suppl 1): 79-85.

48. Bast D, Dresser L, Duncan CL, et al. Short-course therapy of gemifloxacin effective against pneumococcal pneumonia in mice. J Chemother. 2006:18:45-51.

49. Wilson R, Langan C, Ball P, et al; Gemifloxacin 207 Clinical Study Group. Oral gemifloxacin once daily for 5 days compared with sequential therapy with i.v. ceftriaxone/oral cefuroxime (maximum of 10 days) in the treatment of hospitalized patients with acute exacerbations of chronic bronchitis. Respir Med. 2003;97:242-249.

50. Wilson R, Schentag KK, Ball P, Mandell L. 068 Study Group. A comparison of gemifloxacin and clarithromycin in acute exacerbation of chronic bronchitis and long-term clinical outcomes. Clin Ther. 2002:24;639-652.

51. File T, Schlemmer B, Garau J, Lode H, Lynch S, Young C. Gemifloxacin versus amoxicillin/clavulanate in the treatment of acute exacerbations of chronic bronchitis. The 070 Clinical Study group. J Chemother. 2000;12(4):314-325.

52. Ball P, Wilson R, Mandell L, Brown J, Henkel T; 069 Clinical Study Group. Efficacy of gemifloxacin in acute exacerbations of chronic bronchitis: a randomized, double-blind comparison with trovafloxacin. J Chemother. 2001;13:288-298

53. Ball P, File Jr TM, Twynholm M, Henkel T; 061 Study Group. Efficacy and safety of gemifloxacin $320 \mathrm{mg}$ once-daily for 7 days in the treatment of adult lower respiratory tract infections. Int J Antimicrob Agents. 2001;18:19-27.

54. Sethi S, Fogarty C, Fulambarker A. A randomized double-blind study comparing 5 days oral gemifloxacin with 7 days oral levofloxacin in patients with acute exacerbation of chronic bronchitis. Respir Med. 2004;98(8):697-707.

55. Jadad AR, Moore RA, Carroll D, et al. Assessing the quality of reports of randomized clinical trials: is blinding necessary? Control Clin Trials. 1996;17(1):1-12.

56. Frothingham R. Rates of torsades de pointes associated with ciprofloxacin, ofloxacin, levofloxacin, gatifloxacin and moxifloxacin. Pharmacotherapy. 2001;21:1468-1472.

57. Owens RC. QT prolongation with antimicrobial agents: understanding the significance. Drugs. 2004;64(10):1091-1124.

58. Arizona CERT: Center for Education and Research on Therapeutics. QT drug lists by risk groups. April 15, 2009. Accessed on May 11, 2009. Available from: http:/www.azcert.org/medical-pros/drug-lists/ drug-lists.cfm.

59. Falagas ME, Rafailidis PI, Rosmarakis ES. Arrhythmias associated with fluoroquinolone therapy. Int J Antimicrob Agents. 2007;29: 374-379. 
60. US Food and Drug Administration. FDA Black Box Warning for fluoroquinolones. July 8, 2008. Accessed on May 11, 2009. Available from: http://www.fda.gov/cder/drug/InfoSheets/HCP/fluoroquinolonesHCP.htm.
61. Halpern MT, Palmer CS, Zodet M, Kirsch J. Cost-effectiveness of gemifloxacin: results from the GLOBE study. Am J Health Syst Pharm. 2002;59;1357-1365.

\section{Publish your work in this journal}

The International Journal of COPD is an international, peer-reviewed journal of therapeutics and pharmacology focusing on concise rapid reporting of clinical studies and reviews in COPD. Special focus is given to the pathophysiological processes underlying the disease, intervention programs, patient focused education, and self management protocols.
This journal is indexed on PubMed Central, MedLine and CAS. The manuscript management system is completely online and includes a very quick and fair peer-review system, which is all easy to use. Visit http://www.dovepress.com/testimonials.php to read real quotes from published authors. 